\title{
Aprimoramento da Localização de Terminais Móveis utilizando Correlação de Assinaturas de Rádio-Frequência
}

\author{
Rafael Saraiva Campos e Lisandro Lovisolo
}

\begin{abstract}
Resumo-Neste trabalho são propostos aprimoramentos para o método de localização de terminais móveis por correlação de assinaturas de rádio-frequência denominado RF-FING+RTDPRED (Predicted Radio-Frequency Fingerprint with Round Trip Delay). A nova versão foi testada em redes GSM (Global System for Mobile Telecommunications) em uma área urbana densa e em redes $802.11 \mathrm{~b}$ em ambiente indoor. A acurácia obtida após as inovações foi comparada com aquelas alcançadas pela versão original e por um método de posicionamento utilizando redes neurais artificiais (RNA) recentemente proposto. No teste veicular GSM, as inovações propostas resultaram, em relação à versão original, em redução de até $50 \%$ no erro mediano e $47 \%$ no erro máximo. A nova versão do método RF-FING+RTD-PRED atingiu melhor acurácia que o método baseado em RNA nos dois cenários de teste.
\end{abstract}

Palavras-Chave-Localização, Assinaturas de RádioFrequência, Correlação, Modelagem de Propagação.

Abstract-In this paper improvements to the mobile station location method called RF-FING+RTD-PRED are proposed. This method is based on the correlation of radio-frequency fingerprints. Its new version has been tested in a GSM network in a dense urban area and in 802.11b networks in an indoor environment. The attained accuracy after the method's enhancements has been compared to the one achieved by the method's original version and by a recently published positioning method based in artificial neural networks. In the GSM vehicular test, the proposed enhancements resulted, in relation to the original version, in a reduction of $50 \%$ of the median error and $47 \%$ of the maximum error. The new RF-FING+RTD-PRED version achieved better accuracy than the RNA based method in the two test environments.

Keywords-Positioning, Radio-Frequency Fingerprints, Correlation, Propagation Modeling.

\section{INTRODUÇÃO}

A localização de dispositivos móveis em redes sem fio tem recebido crescente atenção, predominantemente devido a: i) exigências de normas reguladoras, particularmente no âmbito da localização de chamadas de emergência originadas em redes de telefonia móvel celular [1][2] e ii) possibilidade de ofertar serviços de valor agregado baseados em localização (LCS - Location Services). Embora haja dispositivos móveis usados em redes sem fio com receptores GPS (Global Positioning System) integrados, o emprego de métodos baseados na rede [3], i.e, que não requerem qualquer modificação ou função específica do terminal, justifica-se principalmente por duas razões: i) os métodos baseados na rede permitem a localização de qualquer terminal móvel (MS - Mobile Station); ii) os métodos baseados na rede podem atuar como fallback, quando métodos mais precisos estiverem

Rafael Saraiva Campos e Lisandro Lovisolo, Faculdade de Engenharia, Programa de Engenharia Eletrônica, Universidade do Estado do Rio de Janeiro, Rio de Janeiro, Brasil, E-mails: rafael_saraiva@ig.com.br, lisandro@uerj.br. indisponíveis devido a limitações sistêmicas - por exemplo, em ambientes indoor ou em áreas urbanas densas, onde a recepção de sinais GPS é muitas vezes inviável.

Os métodos de localização baseados na rede devem usar apenas informações já disponíveis na estação base servidora (BTS - Base Transmission Station) ou fornecidas pelo MS através do relatório de medidas (MR - Measurement Report). Nesta classe de métodos estão incluídos os algoritmos DCM (Database Correlation Methods), que fornecem uma estimativa da posição do MS comparando a assinatura de rádio-frequência (RF) medida com assinaturas de RF armazenadas em um banco de dados de correlação (CDB Correlation Database) [4][5]. Uma assinatura de RF é um conjunto de parâmetros medidos pelo MS e reportados através dos MRs que são periodicamente enviados para a BTS servidora. Parâmetros de RF medidos pela BTS servidora no enlace reverso também podem ser utilizados na composição da assinatura de RF. No CDB, cada assinatura de RF está associada a um par de coordenadas geográficas.

O método RF-FING+RTD-PRED pertence à classe de soluções DCM e foi originalmente proposto em [6]. Ele utiliza assinaturas de rádio frequência (RF-FING - RadioFrequency Fingerprints) obtidas a partir de modelagem de rádio-propagação (PRED - Predicted). Estas assinaturas de RF incluem, além dos níveis de sinal recebido e identidade das estações servidoras, o tempo de propagação entre o MS e a BTS (RTD - Round Trip Delay).

No presente trabalho, inovações foram introduzidas no método RF-FING+RTD-PRED, resultando em significativa redução do erro de localização. Estas inovações incluem a utilização de fatores de ordenação e ponderação em função dos níveis de sinal recebidos, filtragem de média móvel e restrição do espaço de correlação à malha viária (no caso de aplicações veiculares). A acurácia do método foi verificada em redes GSM 850 MHz e 802.11b WiFi (Wireless Fidelity). Em ambos os casos, os resultados foram comparados com aqueles obtidos por um método de localização baseado em RNA [7] recentemente proposto.

A formulação do método RF-FING+RTD-PRED, com o detalhamento das inovações propostas, é feita na Seção II. Os resultados experimentais em redes GSM e WiFi são apresentados na Seção III. As conclusões são apresentadas na Seção IV.

\section{O MÉTODo RF-FING+RTD-PRED}

O método RF-FING+RTD-PRED foi apresentado originalmente em [6], aplicado a redes GSM. Em [8], ele foi generalizado para aplicação em diferentes redes que utilizam multiplexação por divisão de tempo (TDM - Time Division 
Multiplexing) ou de código (CDM - Code Division Multiplexing) na camada física. A acurácia do método RF-FING+RTDPRED foi comparada com a de outros métodos de localização baseados na rede em [6]. Em [8], simulações computacionais avaliaram a acurácia do RF-FING+RTD-PRED em redes WCDMA (Wideband Code Divison Multiple Access).

\section{A. Construção do Banco de Dados de Correlação}

Os CDBs podem ser construídos a partir de medidas de campo ou modelagem de rádio-propagação. Soluções DCM na literatura tipicamente utilizam CBDs construídos a partir de testes de campo [4][9]. A atualização de CDBs utilizados em aplicações LCS em redes WAN (Wide Area Network) sem fio requer extensos testes veiculares que devem ser conduzidos após quaisquer alterações em elementos da rede de acesso rádio. Tais mudanças são constantes em redes de telefonia móvel celular, por exemplo, tornando esta solução impraticável nestes casos. Já a utilização de CDBs construídos a partir de modelagem de rádio-propagação possibilita atualização rápida e previne degradação da acurácia de localização que decorreria do emprego de parâmetros desatualizados no processo de correlação [5].

1) Modelagem de Rádio-Propagação: $\mathrm{O}$ modelo de propagação utilizado para construir o mapa de predição de nível de sinal recebido (RSS - Received Signal Strength) foi o de Okumura-Hata [10], largamente aplicado no planejamento de RF em redes celulares. Nele, a perda de propagação média é calculada por

$$
L=k_{1}+k_{2} \log (d)+k_{3} \log \left(h_{b}\right)+k_{4} L_{f}+k_{5} \log \left(h_{b}\right) \log (d),(1
$$

onde $d$ é a distância em metros entre a BTS e o MS, $h_{b}$ é a altura efetiva da antena da BTS e $L_{f}$ é a atenuação por difração, calculada pelo método de Epstein-Peterson [11]. Assume-se que a altura do MS é igual a 1,5 m. Os coeficientes $k_{i}$ dependem da morfologia e da freqüência de transmissão. Como o teste veicular descrito na Seção III foi realizado em uma rede GSM na banda de $869-881 \mathrm{MHz}$, o modelo foi aplicado na frequência central de $875 \mathrm{MHz}$. Os valores dos coeficientes são $k_{1}=-12,1 ; k_{2}=-44,9 ; k_{3}=-5,83$; $k_{4}=0,5$ e $k_{5}=6,55$. Estes são os valores padrão para o modelo Okumura-Hata em morfologia urbana, exceto $k_{4}$, que foi definido empiricamente pelos autores através de medidas de campo.

2) O Mapa de RSS Predito: A topografia da região de teste é representada por uma matriz $\mathcal{T}=\left(a_{i, j}\right)_{I \times J}$, onde $a_{i, j} \in \Re_{+}$para todo $i \in\{1,2,3, \ldots, I\}$ e $j \in\{1,2,3, \ldots, J\}$. Cada elemento $a_{i, j}$ da matriz armazena a altura de prédios, sobreposta à altura média do terreno em um área quadrada de $r^{2} \mathrm{~m}^{2}$. Se a região de teste cobre uma superfície total de $L \times W$ $\mathrm{m}^{2}$, então $\mathcal{T}$ terá $\left\lceil\frac{L}{r}\right\rceil \times\left\lceil\frac{W}{r}\right\rceil$ elementos, aqui denominados pixels. $\mathrm{O}$ parâmetro $r$ é a resolução planar da matriz $\mathcal{T}$.

A predição de cobertura de uma antena isolada é obtida pela aplicação da Eq. (1) ao perfil do relevo e edificações entre a antena transmissora da BTS e cada pixel na área de teste. As características da antena transmissora - coordenadas geográficas, azimute, diagrama de radiação, potência isotrópica efetivamente irradiada, etc - são consideradas na modelagem de propagação do sinal. O mapa de RSS é obtido pela sobreposição das predições de cobertura de cada uma das antenas transmissoras na área de teste, resultando em uma matriz com $\left\lceil\frac{L}{r^{\prime}}\right\rceil \times\left\lceil\frac{W}{r^{\prime}}\right\rceil \times V$ elementos, onde $V$ é o número de servidores mais fortes considerados em cada pixel, i.e, as células cujos sinais são recebidos com maior intensidade em um dado pixel. O mapa de RSS tem uma resolução planar $r^{\prime}$, onde $r^{\prime} \geq r$. Cada elemento desta matriz armazena o valor de RSS predito e a identidade da célula correspondente. O CDB é construído a partir do mapa de RSS predito.

3) Restrição à Malha Viária: O espaço de correlação ou região de confiabilidade é a área geográfica contendo os pixels candidatos, ou seja, é o conjunto dos elementos da matriz RSS onde é possível que o MS esteja localizado [12]. Para reduzir o espaço de correlação inicial, diminuindo com isso a incerteza final na estimativa de posição, os elementos do mapa de RSS predito podem ser filtrados com base na sua posição. Assim, no caso de aplicações veiculares, é possível restringir o espaço de correlação inicial apenas aos pixels que estejam situados sobre a malha viária. Esta restrição foi aplicada no teste veicular GSM descrito na Seção III.

\section{B. Especificação do Método RF-FING+RTD-PRED}

1) Assinaturas de RF: O algoritmo RF-FING+RTD-PRED obtém a posição estimada do MS através da correlação de uma assinatura de RF medida $\left(\mathrm{RF}_{-} \mathrm{FING}_{M}\right)$ com assinaturas de RF preditas $\left(\right.$ RF-FING $\left._{P}\right)$ armazenadas em um CDB. A assinatura RF-FING ${ }_{M}$ é obtida a partir de um subconjunto dos parâmetros disponíveis no $\mathrm{MR}$ ou na estação servidora. Os parâmetros medidos pelo MS e reportados via MR incluem as identidades da estação servidora e das estações vizinhas e os valores de RSS dos canais de controle destas estações. A medida de RSS é feita no canal de controle porque ele é transmitido com potência constante. O parâmetro RTD é tipicamente medido pela estação servidora em redes que utilizam TDM ou CDM na camada física. Assumindo um tempo de processamento desprezível no MS, o RTD pode ser expresso diretamente em função da distância $l$ percorrida pela onda eletromagnética entre a antena da BTS servidora e o MS [13][14]:

$$
\mathrm{RTD}=\left\lfloor\frac{2 l / c}{\mathrm{~T}_{s}}\right\rfloor
$$

onde $c$ é a velocidade da luz no espaço livre $\mathrm{e}_{s}$ é o período de símbolo.

Cada pixel no mapa de RSS possui uma assinatura RFFING $_{P}$ associada. Os níveis de sinal preditos listados na assinatura RF-FING $_{P}$ são obtidos diretamente dos valores na matriz RSS. O valor de RTD predito $\left(\mathrm{RTD}_{P}\right)$ em cada pixel é calculado pela Eq. (2), assumindo $l$ como a distância de visada direta entre o pixel e a antena da estação melhor servidora predita, que é aquela com maior valor de RSS predito naquele pixel. $\mathrm{O}$ valor de $\mathrm{RTD}_{P}$ pode divergir do valor medido, especialmente devido a condições de propagação sem linha de visada.

Seja $\mathcal{S}$ o conjunto de todos os pixels na área de teste, i.e., os elementos da projeção planar da matriz RSS. A assinatura $\operatorname{RF}-F_{N} G_{P, i}$ do i-ésimo pixel é o par $\left(\operatorname{RTD}_{P, i}, \hat{\nu}_{P, i}\right)$, onde $\hat{\nu}_{P, i}$ é a matriz

$$
\hat{\nu}_{P, i}=\left[\begin{array}{cc}
\operatorname{ID}_{P, 1} & \operatorname{RSS}_{P, 1} \\
\vdots & \vdots \\
\operatorname{ID}_{P, n} & \operatorname{RSS}_{P, n}
\end{array}\right]
$$


A matriz $\hat{\nu}_{P, i}$ tem $n \times 2$ elementos, onde $n$ varia de 1 (quando há apenas uma estação servidora) até $n_{\max }$, que é o número máximo de estações que o MS pode reportar no MR. Cada linha da matriz $\hat{\nu}_{P, i}$ contém a identificação (ID) e o RSS de cada estação monitorada. As linhas são classificadas em ordem decrescente de RSS. A faixa dinâmica de variação do valor de RSS e o passo de quantização deste parâmetro variam em função da tecnologia de acesso sem fio utilizada. Um passo de quantização menor e uma faixa dinâmica maior requerem um MS com maior sensibilidade, o que pode ser utilizado para melhorar a acurácia de localização fornecida pelo método RFFING+RTD-PRED. Em redes GSM, o RSS varia de $-110 \mathrm{dBm}$ até $-48 \mathrm{dBm}$ com um passo de quantização de $1 \mathrm{~dB}$ [15], enquanto em redes 802.16e WiMax (Worldwide Interoperability for Microwave Access) o RSS varia de $-103,75 \mathrm{dBm}$ até $-40 \mathrm{dBm}$ com um passo de quantização de $0,25 \mathrm{~dB}$ [16]. O CDB contendo as assinaturas RF-FING ${ }_{P}$ está completo após o par $\left(\operatorname{RTD}_{P, i}, \hat{\nu}_{P, i}\right)$ ter sido calculado para todos os pixels no conjunto $\mathcal{S}$.

2) Passos de Filtragem para Redução da Região de Confiabilidade e o Processo de Correlação: Para estimar a posição do MS, deve ser realizada a correlação entre a assinatura de RF medida e as assinaturas de RF preditas armazenadas no CDB. Um modo de avaliar a similaridade entre RF-FING R $_{M}$ cada RF-FING ${ }_{P}$ é calcular a distância Euclidiana no espaço $n$-dimensional de entrada [5], onde $n$ é o número de valores de RSS (medidos e preditos) comparados. Poderia assumirse que o MS está localizado no pixel cuja assinatura de RF predita é mais similar à assinatura de RF medida. Porém, esta abordagem levaria a erros significativos devido à imprecisão na medida de RSS pelo MS e à inacurácia inerente à modelagem de propagação. Então, ao invés de considerar apenas um pixel para estimar a posição do MS, os pixels nas $K$ classes mais próximas da assinatura de RF medida são selecionados, e a média de suas coordenadas fornece a estimativa de posição. Em cada classe, todos os pixels têm a mesma distância entre RF-FING $_{M}$ e RF-FING ${ }_{P}$. Porém, não seria praticável realizar este cálculo para todos os pixels na área de teste. Assim, é necessário um processo de filtragem para reduzir o espaço de correlação, sem afetar a acurácia de localização. O método RF-FING+RTD-PRED usa 5 passos de filtragem:

1) Seleção dos pixels em $\mathcal{S}$ que estão situados na área de melhor servidor predita da estação identificada por $\operatorname{ID}_{M, 1}$, obtendo o conjunto $\mathcal{A}=$ $\left\{i \in \mathcal{S} \mid \mathrm{ID}_{P, i, 1}=\operatorname{ID}_{M, 1}\right\}$, onde $\operatorname{ID}_{P, i, 1}$ é a ID do melhor servidor predito no i-ésimo pixel e $\operatorname{ID}_{M, 1}$ é a identidade do melhor servidor medido e reportado via MR. Note que $\mathcal{A} \subset \mathcal{S}$.

2) Seleção dos pixels em $\mathcal{A}$ cujos valores predito e medido de RTD são iguais, obtendo o conjunto $\mathcal{B}=$ $\left\{i \in \mathcal{A} \mid \operatorname{RTD}_{P, i}=\operatorname{RTD}_{M}\right\}$, onde $\operatorname{RTD}_{P, i}$ é o valor de RTD predito no i-ésimo pixel e $\mathrm{RTD}_{M}$ é o valor de RTD medido pela estação servidora. Note que $\mathcal{B} \subset \mathcal{A} \subset \mathcal{S}$.

3) i) Definição do conjunto $\mathcal{F}_{i}$ : este conjunto contém as IDs das estações servidoras listadas na assinatura de RF predita $\left(\right.$ RF-FING $\left._{P, i}\right)$ no i-ésimo pixel e que também estão presentes na assinatura de RF medida $\left(\mathrm{RF}^{-F I N G} \mathrm{G}_{M}\right)$. Assim, se $\mathcal{I}_{P, i}$ é o conjunto de IDs presentes em RF-
FING $_{P}$ no i-ésimo pixel e $\mathcal{I}_{M}$ é o conjunto de IDs presente em RF-FING ${ }_{M}$, então $\mathcal{F}_{i}=\mathcal{I}_{P, i} \cap \mathcal{I}_{M}$; ii) Seleção dos elementos de $\mathcal{B}$ que têm pelo menos $N$ IDs iguais na RF-FING R $_{M}$ e RF-FING $P$, obtendo o conjunto $\mathcal{C}=\left\{i \in \mathcal{B}, N \in\left[1, n_{\text {max }}\right] \mid N \leq \#\left(\mathcal{F}_{i}\right) \leq n_{\max }\right\}$, onde $\#\left(\mathcal{F}_{i}\right)$ é a cardinalidade do conjunto $\mathcal{F}_{i}$. Note que $N \leq n_{\max }$. iii) Se o conjunto $\mathcal{C}$ obtido for vazio, o valor de $N$ é decrementado até que $\mathcal{C}$ tenha pelo menos um elemento. Note que $\mathcal{C} \subset \mathcal{B} \subset \mathcal{A} \subset \mathcal{S}$.

4) Para cada pixel $i \in \mathcal{C}$, distância quadrática entre os valores de RSS medidos $\left(\operatorname{RSS}_{I D_{M, k}}\right)$ e preditos $\left(\operatorname{RSS}_{I D_{P, i, k}}\right)$ é calculado para cada $\operatorname{ID}_{k} \in \mathcal{F}_{i}\left(k \in\left[1, \#\left(\mathcal{F}_{i}\right)\right]\right)$. A distância quadrática total calculada no i-ésimo pixel é dada por:

$d_{i}=\sum_{I D_{k} \in \mathcal{F}_{i}}\left\{\sqrt{f_{I D_{k}} w_{I D_{k}}}\left\lfloor\frac{\mathrm{RSS}_{I D_{M, k}}-\mathrm{RSS}_{I D_{P, i, k}}}{\delta}\right\rfloor\right\}^{2}$

O parâmetro $\delta(\mathrm{dB})$ representa a imprecisão de medida de RSS inerente ao receptor do MS. Os parâmetros $f_{I D_{k}}$ e $w_{I D_{k}}$, descritos a seguir, são o fator de ordenação e o peso, respectivamente.

5) Dentre os pixels pertencentes a $\mathcal{C}$, apenas os pixels nas $K$ classes com as menores distâncias quadráticas serão selecionados, formando o conjunto $\mathcal{D}$. Note que $\mathcal{D} \subset$ $\mathcal{C} \subset \mathcal{B} \subset \mathcal{A} \subset \mathcal{S}$. A posição estimada do MS é a média aritmética das coordenadas dos pixels em $\mathcal{D}$.

3) $O$ Parâmetro de Ponderação $w_{I D_{k}}$ : Para valores de RSS mais baixos a imprecisão na medida torna-se mais significativa, em particular quando o valor de RSS aproximase da sensibilidade do receptor do MS. Logo, a diferença entre os valores de RSS medido e predito deverá ter um maior peso na Eq. (4) para valores de RSS medido mais elevados. Considere o conjunto $\mathcal{F}_{i}$ definido no $3^{\circ}$ passo do processo de correlação. Assuma que as identidades listadas neste conjunto estão classificadas em ordem decrescente dos respectivos valores de RSS medido. Então, $\operatorname{RSS}_{I D_{M, u}} \geq$ $\operatorname{RSS}_{I D_{M, v}}$, se $u \leq v$, onde $u, v \in\left[1, \#\left(\mathcal{F}_{i}\right)\right]$. O peso na escala linear é definido por:

$$
w_{I D_{k}}=10^{0,1 \gamma\left(R S S_{I D_{M, k}}-R S S_{I D_{M, 1}}\right)}
$$

onde $\operatorname{RSS}_{I D_{M, k}}$ é o valor na escala logarítmica do RSS medido do $k$-ésimo elemento do conjunto $\mathcal{F}_{i}$ e $1 \leq \gamma \leq 3$. O parâmetro $\operatorname{RSS}_{I D_{M, 1}}$ corresponde ao valor de RSS medido do melhor servidor reportado em RF-FING $M$. A utilização do peso $w$ equivale a uma variação dinâmica, pixel a pixel, do valor do parâmetro $N$, que é o número mínimo de equivalências entre as identidades dos servidores listados em RF-FING R $_{M} \mathrm{e}$ RF-FING $_{P}$. O efeito da definição de um valor global de $N$ foi analisado em [6].

4) O Fator de Ordenação $f_{I D_{k}}$ : Além da diferença entre os valores de RSS medido e predito, a comparação da sequência das estações servidoras - classificadas em ordem decrescente de RSS - nas assinaturas de RF medida e predita pode ser utilizada para melhorar a correlação e reduzir o erro de localização [17]. O fator de ordenação $f_{I D_{k}}$ multiplica o peso $w_{I D_{k}}$, aumentando-o quando o $k$-ésimo servidor listado em $\mathcal{F}_{i}$ ocupa a mesma posição em RF-FING $_{M}$ e RF-FING $_{P}$, e reduzindo-o quando ocupa posições distintas. Fazemos esta redução proporcional à diferença de posição, assim o fator de ordenação pode ser expresso por:

$$
f_{I D_{k}}=\frac{\mu}{1+\mu\left|m_{k}-p_{k}\right|}
$$


onde $\mu$ é um valor inteiro ajustável e $m_{k}$ e $p_{k}$ são as posições ocupadas pela $k$-ésima estação servidora nas assinaturas RFFING $_{M}$ e RF-FING $P$, respectivamente, sendo $m_{k}, p_{k}, k \in$ $\left[1, \#\left(\mathcal{F}_{i}\right)\right]$. Assume-se que as estações servidoras listadas em $\mathcal{F}_{i}$, RF-FING $_{M}$ e RF-FING ${ }_{P}$ estão classificadas por ordem decrescente de RSS.

5) O Filtro de Média Móvel: Para um dado MS, há uma forte correlação entre suas localizações nos instantes $t_{j}$ e $t_{j}+\Delta t$, desde que o produto da velocidade $v$ do $\mathrm{MS}$ e do intervalo de tempo $\Delta t$ seja pequeno [18]. O filtro de média móvel atua como um filtro passa-baixa, suavizando desvios entre estimativas de localização subsequentes. Utilizando um filtro de comprimento $L$, as coordenadas estimadas $\left(\hat{x}_{j}, \hat{y}_{j}\right)$ do MS no instante $t_{j}$ serão dadas pela média aritmética das coordenadas da estimativa atual e das $L-1$ anteriores, ou seja:

$$
\hat{x}_{j}=\frac{1}{L} \sum_{u \in[0, L-1]} \hat{x}_{j-u} \quad \hat{y}_{j}=\frac{1}{L} \sum_{u \in[0, L-1]} \hat{y}_{j-u}
$$

O valor ótimo de $L$ depende do produto $v \cdot \Delta t$ e das variações de direção e velocidade do MS [18].

\section{AVAliaçÃo EXPERIMENTAL}

\section{A. Cenários de Teste}

1) GSM Outdoor: Foram coletados $360 \mathrm{MRs}$ ao longo da Av. Presidente Vargas, no Centro da cidade do Rio de Janeiro, tendo sido detectadas 32 estações servidoras na rota de 1, 03 $\mathrm{km}$. O conjunto de teste era composto de um celular GSM e um receptor GPS, ambos conectados a um laptop instalado no interior de um veículo. O MS estava em modo ativo e para cada MR transmitido (1 a cada $480 \mathrm{~ms}$ ) o valor de RTD foi identificado e a posição do MS calculada via GPS. Cada MR registra a identidade e o valor de RSS da estação servidora e de até 6 estações vizinhas. A localização fornecida pelo GPS foi tomada como referência. Assim, para cada MR, o erro de posicionamento é a distância Euclidiana entre a posição dada pelo GPS e a posição estimada pelo método RF-FING+RTDPRED. Para a correlação, foi utilizado um CDB construído a partir de um mapa de RSS predito com resolução planar $r^{\prime}=10 \mathrm{~m}$. O mapa de RSS foi calculado sobre uma base topográfica digitalizada com resolução planar $r=5 \mathrm{~m}$ e contendo altimetria dos prédios. O espaço de correlação foi restrito à malha viária, conforme descrito na Seção II-A.3.

2) Wi-Fi Indoor: Foram feitas medidas em 262 posições com espaçamento de aproximadamente 3 metros nos corredores do $4^{\circ}$ andar do prédio da UERJ (Universidade do Estado do Rio de Janeiro). O conjunto de medição era composto de um laptop com placa WiFi Atheros. O software NetStumbler foi utilizado para registrar os valores de RSS por AP em intervalos de 1 segundo. Em cada posição de medida foram calculados os valores médios de RSS por AP em intervalos de 90 a 120 segundos. Estes valores médios foram usados na construção do CDB. A utilização de um CDB baseado em medidas se justifica no caso de sistemas de localização indoor pois sua atualização é mais simples e a modelagem de rádio-propagação é mais complexa [19]. Para o teste de localização foram empregados os valores médios de RSS por AP calculados em intervalos de 5 segundos em cada uma das posições de medição. Tempos de integração mais elevados seriam incompatíveis com a condição que se deseja simular, que é a de um usuário WiFi deslocando-se ao longo dos corredores. Em cada posição de medição, apenas os 7 APs recebidos com maior intensidade foram utilizados na composição da assinatura de RF medida.

\section{B. Testes na Rede GSM Outdoor}

1) Avaliação do Ganho de Acurácia Resultante das Inovações no Método RF-FING+RTD-PRED: As modificações introduzidas no método RF-FING+RTD-PRED em relação a [6] foram: i) restrição do espaço de correlação à malha viária; ii) utilização de um filtro de média móvel; iii) inserção de um fator de ponderação; iv) inserção de um fator de ordenação. Os melhores resultados foram obtidos para $L=20, \mu=100$ e $\gamma=1,5$. A imprecisão na medida de RSS feita por um terminal GSM padrão, expressa pelo parâmetro $\delta$, é igual a $6 \mathrm{~dB}$ [15]. A Fig. 1 ilustra a rota do teste GSM outdoor e as estimativas de posição fornecidas pelo método RF-FING+RTD-PRED antes e depois das modificações. Observa-se que estas estimativas, antes agrupadas em 3 clusters, passam a acompanhar a trajetória do MS. O efeito dos aprimoramentos introduzidos é descrito na Tab. I, onde o estágio $I$ considera restrição à malha viária; o estágio $I I$ equivale ao estágio $I$ acrescido de ponderação e ordenação; o estágio $I I I$ equivale ao estágio $I$ acrescido de filtro de média móvel e a versão final equivale ao estágio $I I I$ acrescido de ponderação e ordenação. Para a versão final, há uma redução de até $50 \%$ no erro mediano e $47 \%$ no erro máximo. O gráfico da distribuição de probabilidade cumulativa (CDF Cummulative Distribution Function) das versões original e final é ilustrado na Fig. 4. Os resultados obtidos sugerem que o método proposto pode ser usado para o rastreamento de veículos.

TABELA I

ERRO DE LOCALIZAÇÃo EM METROS NO TESTE EM REDE GSM outdoor.

\begin{tabular}{|l||c|c|c|c|}
\hline \multicolumn{1}{|c||}{} & \multicolumn{4}{c|}{ Percentis } \\
\hline RF-FING+RTD-PRED & $50 \%$ & $67 \%$ & $95 \%$ & $100 \%$ \\
\hline \hline Versão Original & 99 & 156 & 336 & 408 \\
\hline Estágio I & 68 & 124 & 264 & 331 \\
\hline Estágio $I I$ & 67 & 100 & 227 & 276 \\
\hline Estágio III & 58 & 113 & 229 & 243 \\
\hline Versão Final & 50 & 78 & 203 & 219 \\
\hline
\end{tabular}

2) Comparação do Método RF-FING+RTD-PRED com Método de Localização Utilizando RNA: A acurácia do método RF-FING+RTD-PRED foi comparada com a de um método de localização baseado em uma RNA do tipo backpropagation [7]. As assinaturas de RF com parâmetros normalizados são os vetores de entrada e as coordenadas estimadas a saída da RNA. A normalização tem como objetivo adequar os valores de entrada à faixa de valores de saída da função de ativação dos 15 neurônios da camada escondida [7]. Para permitir comparação direta entre os métodos, o mesmo CDB utilizado pelo método RF-FING+RTD-PRED foi empregado para o treinamento da RNA. Na fase de teste foram utilizados os 360 MRs coletados conforme descrito na Seção III-A.1. As 


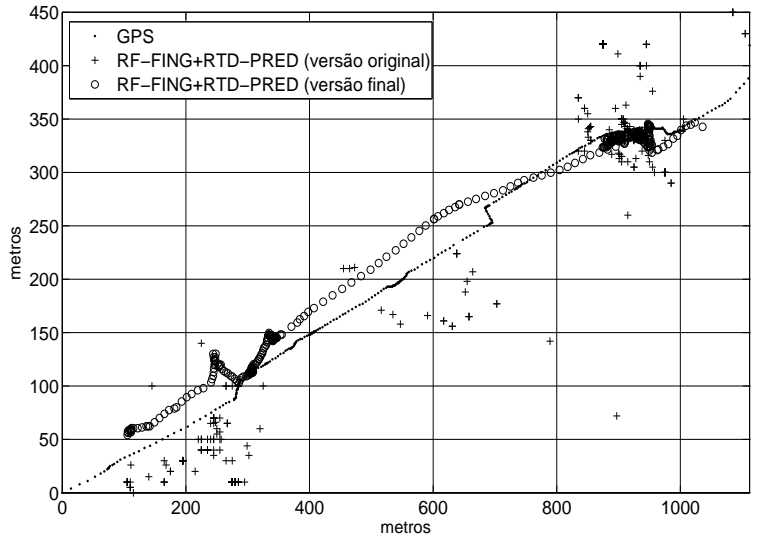

Fig. 1. Rota do teste GSM outdoor e estimativas de localização dadas pelo método RF-FING+RTD-PRED antes e depois das modificações.

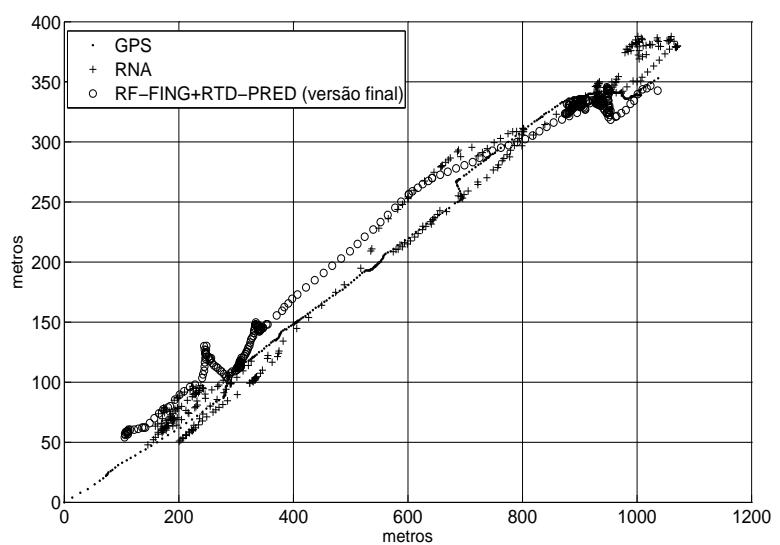

Fig. 2. Rota do teste GSM outdoor e estimativas de localização dadas pela RNA e pelo método RF-FING+RTD-PRED depois das modificações.

posições estimadas pelos dois métodos foram fornecidas a um filtro de média móvel do tipo descrito na Seção II-B.5 com comprimento $L=20$. Nestas condições, o método baseado na RNA produz estimativas de posição com desvios de 95 , 131, 277 e 314 metros para os percentis $50 \%, 67 \%, 95 \%$ e $100 \%$. Na Tab. I verifica-se que a versão final do método RFFING+RTD-PRED tem acurácia superior, com desvios de 50, 78, 203 e 219 metros para os mesmos percentis. A Fig. 2 ilustra a rota de teste e as posições estimadas pelo método RF-FING+RTD-PRED e pela RNA. A Fig. 3 exibe o gráfico em coordenadas polares do erro de localização obtido com a RNA e com a versão final do método RF-FING+RTD-PRED. O erro está angularmente distribuído ao longo do eixo da rota de teste em ambos os casos, uma vez que utilizou-se restrição à malha viária, porém a dispersão alcançada pelo método proposto é inferior. Isto é confirmado pela Fig. 4, que mostra a CDF do erro de localização fornecido pela RNA e pelo método RF-FING+RTD-PRED antes e após as modificações. Observa-se que a acurácia da localização com a RNA é muito próxima àquela obtida com a versão original do método RF-FING+RTD-PRED. Já a versão final do método

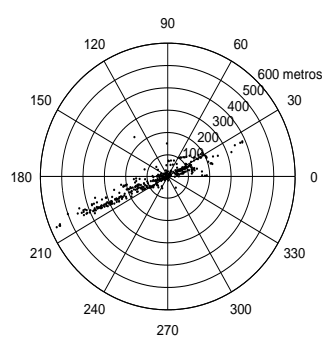

(a)

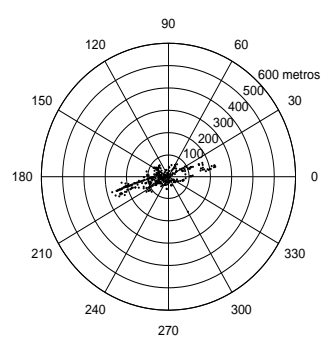

(b)
Fig. 3. Erro de localização da RNA (a) e da versão final do método RFFING+RTD-PRED (b) no teste GSM outdoor.

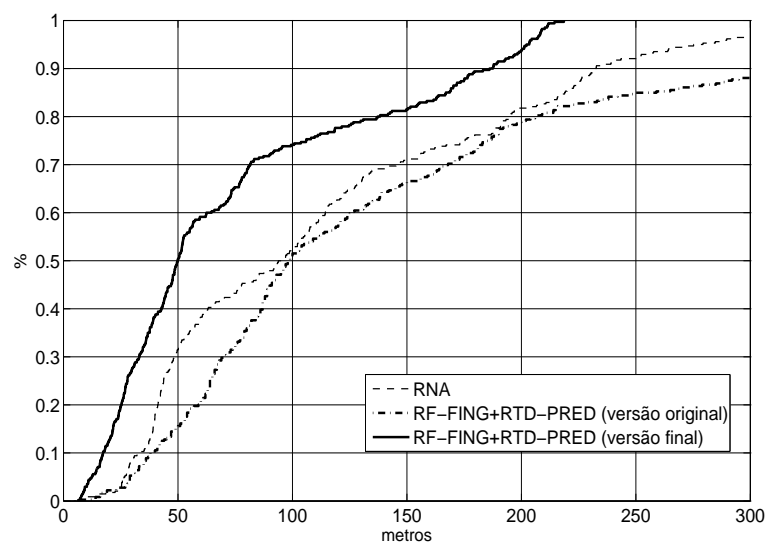

Fig. 4. CDF do erro de localização da RNA e do método RF-FING+RTDPRED no teste GSM outdoor, antes e depois das modificações.

RF-FING+RTD-PRED tem um melhor desempenho, com um erro mediano quase $50 \%$ inferior.

\section{Testes na Rede Wi-Fi Indoor}

1) Comparação do Método RF-FING+RTD-PRED com Método de Localização Utilizando RNA: A mesma RNA citada na Seção III-B.2 foi empregada nesta comparação. Para o método RF-FING+RTD-PRED utilizou-se $\mu=100, \gamma=1,5$ e $\delta=5 \mathrm{~dB}$ [20]. Não foi aplicado o filtro de média móvel. O CDB utilizado para correlação no método RF-FING+RTDPRED e para o treinamento da RNA foi construído a partir de medidas, conforme procedimento descrito na Seção IIIA.2. As posições de medição e as localizações estimadas pelos dois métodos são exibidas na Fig 5. Observa-se que o método RF-FING+RTD-PRED produz dispersão muito menor, o que é confirmado pela análise da CDF do erro na Fig 6. A Tab. II lista os valores de erro de localização para diferentes percentis. Nota-se que o erro mediano do método RF-FING+RTD-PRED é igual ao espaçamento entre as posições de medição.

\section{CONCLUSÕES}

Neste trabalho foram apresentados aprimoramentos do método de localização RF-FING+RTD-PRED, anteriormente proposto, que resultaram em significativa redução do erro 
de localização. A acurácia alcançada pela nova versão foi comparada com a da versão original e com a de um método de localização baseado em redes neurais artificiais recentemente publicado. Os resultados obtidos no teste outdoor em área urbana densa sugerem que o método RF-FING+RTD-PRED pode ser empregado para o rastreamento de veículos, mesmo com a utilização de um CDB baseado em modelagem de propagação. Os resultados obtidos no teste indoor mostraram que o método RF-FING+RTD-PRED pode alcançar uma acurácia média próxima à distância entre os pontos de medição utilizados na composição do CDB.

\section{TABELA II}

ERRo de LOCALIZAÇÃo EM METROS NO TESTE EM REDES WIFI indoor.

\begin{tabular}{|l||c|c|c|c|}
\hline \multicolumn{1}{|c||}{} & \multicolumn{4}{c|}{ Percentis } \\
\hline Método & $50 \%$ & $67 \%$ & $95 \%$ & $100 \%$ \\
\hline \hline RF-F ING+RTD-PRED & 3 & 5 & 28 & 113 \\
\hline RNA & 16 & 25 & 66 & 113 \\
\hline
\end{tabular}

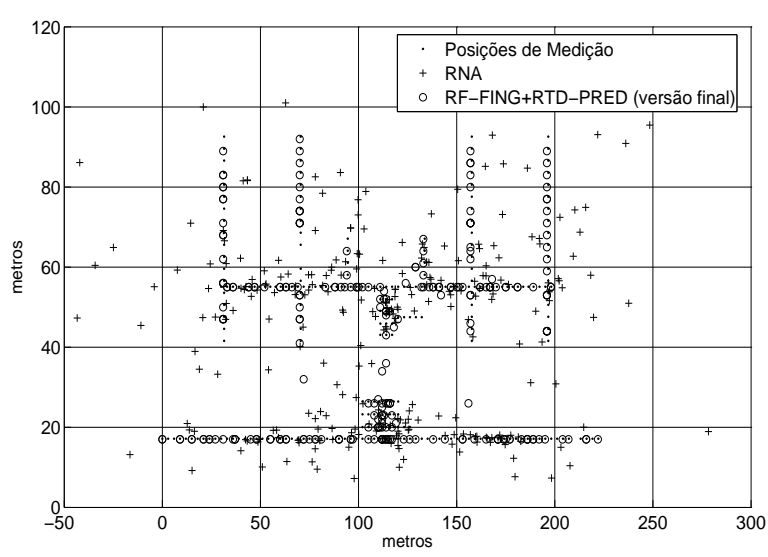

Fig. 5. Posições de medição no teste WiFi indoor e estimativas de localização.

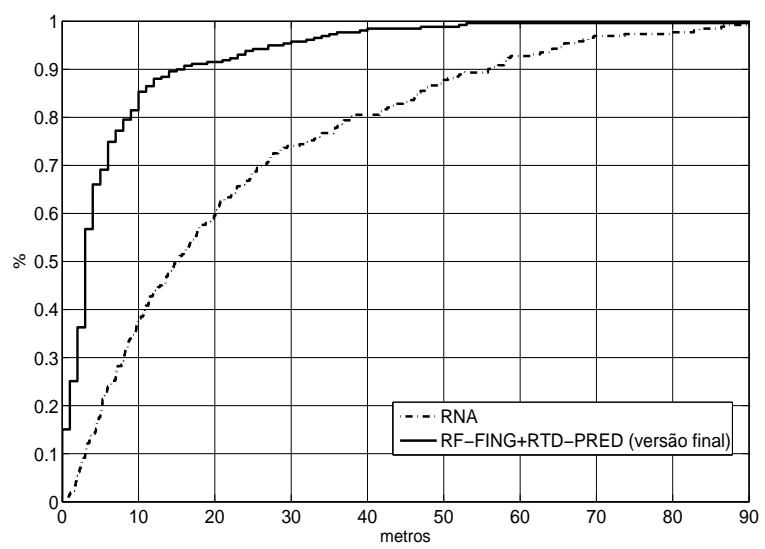

Fig. 6. CDF do erro de localização no teste WiFi indoor.

\section{REFERÊNCIAS}

[1] FCC, FCC Wireless Enhanced E911 Requirements. USA, 1999.

[2] European Commission, "Commission Recommendation on the processing of caller location information in electronic communication networks for the purpose of location-enhanced emergency call services 2003/558/EC", Official Journal of the European Union, Julho 2003.

[3] M. A. Spirito e S. Pöykko e O. Knuuttila, "Experimental Performance of Methods to Estimate the Location of legacy Handsets in GSM", Proc. of IEEE Vehicular Tech. Conf., 2001.

[4] M. Chen et. al., "Practical Metropolitan-scale Positioning for GSM Phones", Proc. of 8th Ubiquitous Computing International Conference, 2006.

[5] H. Laitinen et. al, "Database Correlation Method for GSM Location", Proc. of IEEE 53rd Vehicular Technology Conference, 2001.

[6] R. S. Campos e L. Lovisolo, "Análise Empírica de Métodos de Localização de Terminais GSM Legados utilizando Predição de Cobertura e Alinhamento Temporal", XXVI Simpósio Brasileiro de Telecomunicações, 2008.

[7] M. A. Spirito et. al., "WLAN-Based Real Time Vehicle Locating System", Proc. of IEEE 69th Vehicular Technology Conference, 2009.

[8] R. S. Campos e L. Lovisolo, "A Fast Database Correlation Algorithm for Localization of Wireless Network Mobile Nodes using Coverage Prediction and Round Trip Delay", Proc. of IEEE 69th Vehicular Technology Conference, 2009.

[9] M. Al Hallak, M.S. Safadi e R. Kouatly, "Mobile Positioning Technique using Signal Strength Measurement method with the aid of Passive Mobile Listener Grid", Proc. of 2nd International Conference on Information and Communication Technologies, 2006.

[10] M. Hata, "Empirical Formula for Propagation Loss in Land Mobile Radio Services", IEEE Transactions on Vehicular Technology, vol. 29, no. 3, Sep. 1980.

[11] M. D. Yacoub, Foundations of Mobile Radio Engineering. CRC Press, 1993.

[12] M. Weckström e M. Spirito e V. Ruutuu, "Mobile Station Location", GSM, GPRS and EDGE Performance. John Wiley and Sons, 2002, pp. $119-141$.

[13] European Telecommunications Standard Institute, "ETSI TS 100912 v8.12.0 (2003-08) - Digital cellular telecommunications system (Phase $2+$ ); Radio subsystem synchronization (3GPP TS 05.10 version 8.12.0 Release 1999)", 2003.

[14] 3GPP TS 25214, v5.0.0 (2002-03), "3rd Generation Partnership Project; Technical Specification Group Radio Access Network; Physical Layer Procedures (FDD) (Release 5)", 2002.

[15] ETSI TS 100911 V6.2.0 (1998-07), "Digital cellular telecommunications system (Phase 2+); Radio subsystem link control (GSM 05.08 version 6.2.0 Release 1997)", 1998.

[16] IEEE Computer Society and IEEE Microwave Theory and Techniques Society, "IEEE Standard for Local and Metropolitan Area Networks; Part 16: Air Interface for Fixed and Mobile Broadband Wireless Access Systems; Amendment 2: Physical and Medium Access Control Layers for Combined Fixed and Mobile Operation in Licensed Bands", 2006.

[17] Y. Chenghung et. al.,"Accuracy characterization for metropolitan-scale Wi-Fi localization", MobiSys '05: Proc. of the 3rd international conference on Mobile systems, applications, and services, 2005.

[18] X. Shen, J. W. Mark e J.Ye, "Mobile Location Estimation in CDMA Cellular Networks by Using Fuzzy Logic", Wireless Personal Communications, no. 22, 2002.

[19] P. Bahl e V. N. Padmanabhan, "RADAR: An In-Building RF-based User Location and Tracking System", INFOCOM 2000: Proc. of the 19th Annual Joint Conference of the IEEE Computer and Communications Societies, 2000.

[20] IEEE Computer Society, "IEEE Standard for Information Technology Telecommunications and informaton exchange between systems - Local and metropolitan area networks - Specific requirements; Part 11: Wireless LAN Medium Access Control (MAC) and Physical Layer (PHY) Specifications", 2007. 